\title{
COVID-19 infection in hematopoietic cell transplantation: age, time from transplant and steroids matter
}

\author{
Ankur Varma $\mathbb{D}^{1} \cdot$ Satyajit Kosuri ${ }^{2} \cdot$ Celalettin Ustun $\mathbb{D}^{1} \cdot$ Uroosa Ibrahim $\mathbb{D}^{3}$ - Jonathan Moreira ${ }^{4}$. \\ Michael R. Bishop ${ }^{2}$ Sunita Nathan ${ }^{1} \cdot$ Jayesh Mehta ${ }^{4} \cdot$ Daniel Moncayo $^{3} \cdot$ Joseph Heng $^{2} \cdot$ Keren Osman $^{3}$. \\ Kehinde U. A. Adekola ${ }^{4}$
}

Received: 22 July 2020 / Revised: 29 July 2020 / Accepted: 5 August 2020 / Published online: 14 August 2020

(c) Springer Nature Limited 2020

\section{To the Editor:}

We read with great interest the paper by He et al. [1] on the outcome of COVID-19 infection in hematological malignancy patients. This study was one of the first to report the outcome in hematological malignancy patients and since then many others have reported theirs [2-5]. Most of these studies $[1-4,6]$ included none or very few hematopoietic stem cell transplant $(\mathrm{HCT})$ recipients $(N<4)$ and therefore we like to present the largest data to our knowledge of the impact of COVID 19 infection in HCT recipients. We identified $34 \mathrm{HCT}$ recipients [autologous, (autoHCT) $N=14$ and allogeneic, (alloHCT) $N=20$ ] diagnosed with COVID19 across 4 cancer centers (Rush University, The University of Chicago, Mount Sinai and Northwestern University) in Chicago and New York City.

The median age for all patients was 57 years; 54 in alloHCT and 59 years in the autoHCT group (Table 1). The

These authors contributed equally: Ankur Varma, Celalettin Ustun

Supplementary information The online version of this article (https:// doi.org/10.1038/s41375-020-01019-x) contains supplementary material, which is available to authorized users.

Ankur Varma

Ankur_Varma@rush.edu

1 Department of Medicine, Division of hematology, Oncology and Cell Therapy, The Coleman Foundation Blood and Marrow Transplant Center at Rush University Medical Center, Chicago, IL, USA

2 Department of Medicine, Section of Hematology/Oncology, The University of Chicago, Chicago, IL, USA

3 Bone Marrow Transplant and Cellular Therapy Program, Tisch Cancer Institute, Mount Sinai Hospital, New York, NY, USA

4 Robert H. Lurie Comprehensive Cancer Center, Feinberg School of Medicine, Northwestern University, Chicago, IL, USA majority of patients were Caucasian males. Eleven patients $(32 \%)$ were obese (BMI $\geq 30)$ and $20(59 \%)$ had a history of smoking. Hypertension $(N=15,44 \%)$ and diabetes $(N=$ $13,38 \%)$ were the most common comorbidities among all patients. Acute leukemia $(N=16,47 \%)$, followed by multiple myeloma $(N=9,26 \%)$ and lymphoma $(N=6,18 \%)$ were the most common diagnoses. Peripheral blood $(N=$ $30,88 \%$ ) was the stem cell source for the majority of them and most of the patients received myeloablative conditioning $(N=21,62 \%)$.

The median time after HCT at which a COVID-19 infection occurred was 17.4 months for all patients; 18.9 months in the alloHCT and 13.2 months in the autoHCT recipients. Nine patients (26\%) had active acute or chronic graft-versus-host disease (GVHD) at the time of COVID-19; 15 (44\%) were on immunosuppressive drugs (ISP). Eleven (32\%) patients were on steroids; ten (50\%) in alloHCT (nine for GVHD, one for adrenal insufficiency), and one $(7 \%)$ in autoHCT for relapse. The most common COVID-19 presenting symptom was fever $(N=25,74 \%)$, followed by cough $(N=24,71 \%)$ and dyspnea $(N=20$, $59 \%)$ (Table 1). Lymphopenia $(N=12 / 30,40 \%)$, anemia $(N=18 / 30,60 \%)$ and thrombocytopenia $(N=11 / 30,37 \%)$ were common. D-dimer was elevated in $11 / 19$ patients (58\%); 10/13 alloHCT patients (77\%) and 1/6 (17\%) autoHCT patients. Chest radiography obtained in 28 patients showed that bilateral infiltrates $(N=21,75 \%)$ was the most common abnormality.

Twenty patients (59\%) had mild/moderate and 14 (41\%) had severe COVID-19 at presentation. Twenty-five of 34 patients (74\%) [alloHCT $N=14,41 \%$; autoHCT $N=11$, $32 \%$ ] were hospitalized for COVID-19 (Table 2). The median duration of hospitalization was 8 days (range, 2-50 days). Eleven patients (32\%) [alloHCT $N=8,24 \%$; autoHCT $N=3,9 \%$ ] were transferred to the medical intensive care unit (MICU). Eight of these patients (73\%) were intubated [alloHCT group $N=6$, autoHCT group $N=2$ ], 
Table 1 Patient and transplant characteristics and clinical features at presentation.

\begin{tabular}{|c|c|c|c|}
\hline Variables & $\begin{array}{l}\text { Autologous HCT } \\
N=14(41 \%)\end{array}$ & $\begin{array}{l}\text { Allogeneic HCT } \\
N=20(59 \%)\end{array}$ & $\begin{array}{l}\text { Total } \\
N=34(\%)\end{array}$ \\
\hline \multicolumn{4}{|l|}{ Patient characteristics } \\
\hline Age in years (median; range) & $59 ; 29-76$ & $54 ; 24-75$ & $57 ; 24-76$ \\
\hline Gender (F/M) & $3(21) / 11(79)$ & $9(45) / 11(55)$ & $12(35) / 22(65)$ \\
\hline Ethnicity (W/AA/His/Other) & $4(29) / 5(36) / 2(14) / 3(21)$ & $8(40) / 4(20) / 7(35) / 1(5)$ & $12(35) / 9(26.5) / 9(26.5) / 4(12)$ \\
\hline $\mathrm{BMI}(\leq 20 / \geq 30 />40)$ & $2(14) / 7(50) / 2(14)$ & $4(20) / 4(20) / 0$ & $6(18) / 11(32) / 2(6)$ \\
\hline History of smoking & $9(64)$ & $11(55)$ & $20(59)$ \\
\hline History of alcohol use & $2(14)$ & $8(40)$ & $10(29)$ \\
\hline HTN & $8(57)$ & $7(35)$ & $15(44)$ \\
\hline Diabetes & $5(36)$ & $8(40)$ & $13(38)$ \\
\hline CAD & $1(7)$ & $2(10)$ & $3(9)$ \\
\hline $\mathrm{CHF}$ & 0 & $2(10)$ & $2(6)$ \\
\hline COPD & 0 & $3(15)$ & $3(9)$ \\
\hline History of DVT & $1(7)$ & $4(20)$ & $5(15)$ \\
\hline History of PE & $1(7)$ & $3(15)$ & $4(12)$ \\
\hline \multicolumn{4}{|l|}{ Transplant characteristics } \\
\hline Disease (AL/Lym/MM/AA/GCT) & 0/6 (43)/7 (50)/0/1 (7) & $16(80) / 0 / 2(10) / 2(10) / 0$ & $16(47) / 6(18) / 9(26) / 2(6) / 1(3)$ \\
\hline Disease status at transplant $(\mathrm{CR} \pm)$ & $6(43) / 8(57)$ & $14(70) / 6(30)$ & $20(60) / 14(40)$ \\
\hline HCT CI $(>3)$ & $5(36)$ & $5(25)$ & $10(30)$ \\
\hline KPS $(<90 \%)$ & $8(57)$ & $6(30)$ & $14(41)$ \\
\hline SC source (BM or UC) & 0 & $4(20)$ & $4(12)$ \\
\hline Conditioning regimen (MAC vs RIC/NMA) & $14(100) / 0$ & $7(35) / 13(65)$ & $21(62) / 13(38)$ \\
\hline History of Acute GVHD & - & $9(45)$ & $9(26)$ \\
\hline History of Chronic GVHD & - & $8(40)$ & $8(24)$ \\
\hline \multicolumn{4}{|c|}{ Characteristics at diagnosis of COVID-19 infection $N(\%)$} \\
\hline $\begin{array}{l}\text { Time from Transplant in months } \\
\text { (median; range) }\end{array}$ & $13.2 ; 1-178$ & $18.9 ; 1-248.7$ & $17.4 ; 1-248.7$ \\
\hline Active smoker & $1(7)$ & $1(5)$ & $2(6)$ \\
\hline Active GVHD acute/chronic & - & $3(15) / 6(30)$ & $9(26)$ \\
\hline Active ISP at diagnosis & $1(7)$ & $14(70)$ & $15(44)$ \\
\hline Active steroids at diagnosis & $1(7)$ & $10(50)$ & $11(32)$ \\
\hline \multicolumn{4}{|l|}{ Clinical features at diagnosis $N(\%)$} \\
\hline Fever & $10(71)$ & $15(75)$ & $25(74)$ \\
\hline Cough & $11(79)$ & $13(65)$ & $24(71)$ \\
\hline SOB & $9(64)$ & $11(55)$ & $20(59)$ \\
\hline Anosmia/Loss of taste & $1(7)$ & $3(15)$ & $4(12)$ \\
\hline Myalgia & $3(21)$ & $5(25)$ & $8(24)$ \\
\hline Headache & $3(21)$ & $1(5)$ & $4(12)$ \\
\hline Sore throat & $2(14)$ & $2(10)$ & $4(12)$ \\
\hline $\mathrm{N} / \mathrm{V}$ & $2(14)$ & $3(15)$ & $5(15)$ \\
\hline Diarrhea & $4(29)$ & $3(15)$ & $7(21)$ \\
\hline \multicolumn{4}{|c|}{ Laboratory results at diagnosis of COVID-19 infection: median (range). $N^{\mathrm{a}}$} \\
\hline $\mathrm{WBC}\left(\times 10^{9} / \mathrm{L}\right)$ & $5.3(2.3-11.3)$ & $5.9(1.7-13.72)$ & $5.85(1.7-13.72)$ \\
\hline $\mathrm{ANC}\left(\times 10^{9} / \mathrm{L}\right)$ & $3.2(1.69-8.5)$ & $4.03(1.15-11.49)$ & $3.945(1.15-11.49)$ \\
\hline $\operatorname{ALC}\left(\times 10^{9} / \mathrm{L}\right)$ & $0.9(0.2-1.6)$ & $1.1(0.2-37.7)$ & $1(0.2-3)$ \\
\hline Hemoglobin $\mathrm{g} / \mathrm{dL}$ & $12.1(9.3-15.6)$ & $10.9(7-14.8)$ & $11.3(7-15.6)$ \\
\hline Platelet $\left(\times 10^{9} / \mathrm{L}\right)$ & $156(71-299)$ & $164(19-389)$ & $162.5(19-389)$ \\
\hline
\end{tabular}


Table 1 (continued)

\begin{tabular}{llll}
\hline Variables & $\begin{array}{l}\text { Autologous HCT } \\
N=14(41 \%)\end{array}$ & $\begin{array}{l}\text { Allogeneic HCT } \\
N=20(59 \%)\end{array}$ & $\begin{array}{l}\text { Total } \\
N=34(\%)\end{array}$ \\
\hline BUN mg/dL & $22(8-39)$ & $17(6-35)$ & $17.5(6-39)$ \\
Creatinine mg/dL & $1.28(0.59-3.0)$ & $1.0(0.4-2.38)$ & $1.01(0.4-3.0)$ \\
CPK U/L, $N=11$ & $131(46-773)$ & $22(11-111)$ & $43.5(11-773)$ \\
Fibrinogen $\mathrm{mg} / \mathrm{dL}, N=12$ & $642(339-725)$ & $454(289-1009)$ & $467(289-1009)$ \\
D-dimer $\mathrm{mg} / \mathrm{L}, N=19$ & $0.3(0.15-0.77)$ & $1.3(0.18-3.8)$ & $0.7(0.15-3.8)$ \\
Ferritin $\mathrm{ng} / \mathrm{Ml}, N=19$ & $1449(308-2531)$ & $5432(32-13851)$ & $2133.5(32-13851)$ \\
CRP mg/L, $N=18$ & $55.7(3-229.9)$ & $27.95(0.9-310)$ & $32(0.9-310)$ \\
LDH U/L, $N=26$ & $369(211-560)$ & $296.5(129-578)$ & $303(129-578)$ \\
Chest radiography (Bil infiltrates, Pl eff), & $10(71)$ & $13(65)$ & $23(68)$ \\
$N=28$ & & & \\
\hline
\end{tabular}

${ }^{\mathrm{a}} N=30$, unless specified otherwise.

$A L$ acute leukemia, $A L C$ absolute lymphocyte count, $A N C$ absolute neutrophil count, $A A$ African American, $A A$ aplastic anemia, $B i l$ bilateral, $B M I$ body mass index, $B M$ bone marrow, $B U N$ blood urea nitrogen, $C A D$ coronary artery disease, $C H F$ congestive heart failure, $C R$ complete response, $C O P D$ chronic obstructive pulmonary disease, $C P K$ creatine phosphokinase, $C R P$ C-reactive protein, $D M$ diabetes mellitus, $D V T$ deep venous thrombosis, $F$ female, $G C T$ germ cell tumor, $G V H D$ graft versus host disease, $H C T$ hematopoietic stem cell transplant, $H C T$ - $C I$ hematopoietic cell transplantation-specific comorbidity index, $H b$ hemoglobin; His Hispanics, HTN hypertension; ISP immunosuppression; LDH lactate dehydrogenase; KPS Karnofsky Performance Status, Lym lymphoma, $M$ male, MICU medical intensive care unit, $M M$ multiple myeloma, $M A C$ myeloablative conditioning, $N / V$ nausea/vomiting, $N M A$ non myeloablative conditioning, $P E$ pulmonary embolism, $P L T$ platelets, $P l$ eff pleural effusion, $R I C$ reduced intensity conditioning, $S C$ stem cell, $U C$ Umbilical cord, $W$ White, WBC white blood cell.

Table 2 Hospital course of the admitted pts $(N=25)$.

\begin{tabular}{lc}
\hline Hospitalization course & Total No. of patients \\
\hline Admitted, $N(\%)$ & $25(74 \%)$ \\
Allogeneic, $N(\%)$ & $14(41 \%)$ \\
$\quad$ Autologous, $N(\%)$ & $11(32 \%)$ \\
a Duration of Hospitalization, median days & $8(2-50)$ \\
(range) & $13.5(2-50)$ \\
Allogeneic, median days (range) & $6(3-29)$ \\
Autologous, median days (range) & $11(32 \%)$ \\
Medical Intensive care unit, $N(\%)$ & $8(24 \%)$ \\
$\quad$ Allogeneic, $N(\%)$ & $3(9 \%)$ \\
Autologous, $N(\%)$ & $8(24 \%)$ \\
Intubation, $N(\%)$ & $6(18 \%)$ \\
$\quad$ Allogeneic, $N(\%)$ & $2(6 \%)$ \\
Autologous, $N(\%)$ & $2(6 \%)$ \\
Hemodialysis, $N(\%)$ & $7(21 \%)$ \\
Death, $N(\%)$ & $15(7-42)$ \\
Time to death from COVID-19 dx, median & $11(7-42)$ \\
days (range) & $19.5(15-24)$ \\
Allogeneic, median days (range) & \\
Autologous, median days (range) &
\end{tabular}

${ }^{\text {a }}$ Thirty-three pts had one hospitalization, one had three hospitalization, and one another patient had two hospitalizations for treatment of COVID-19 and its complications. For the pts who had $>1$ hospitalization, their duration of hospitalization is the sum of all hospitalizations.

$N$ number; $d x$ diagnosis; $p t s$ patients. and two needed hemodialysis. Hydroxychloroquine $(N=15$, $44 \%)$ was the most frequently administered therapy for COVID-19, followed by Tocilizumab $(N=6,18 \%)$, Remdesivir $(N=5,15 \%)$, convalescent plasma $(N=2)$, LopinavirRitonavir $(\mathrm{N}=1)$, Anakinra $(\mathrm{N}=1)$, Sarilumab $(N=1)$, and Ribavirin $(N=1)$. None of the patients had their ISP discontinued, one hospitalized patient had tacrolimus dose reduced due to renal dysfunction. Seven patients $(21 \%)$ died [alloHCT $N=5$, autoHCT $N=2$ ] at a median of 15 days from their COVID-19 diagnosis (range, 7-42); six of the 7 had severe COVID-19 at presentation and one had mild/moderate disease. The mortality rates among the mild/moderate and severe groups were 5\% $(N=1 / 20)$ and $43 \%(N=6)$, respectively (Supplementary Figs. 1-3).

Analyzing the factors (Supplementary Table 1) associated with MICU admission and mortality, we found that age had a definitive impact on outcomes (Supplementary Fig. 4). For the entire cohort, patients $<40$ years avoided MICU transfer or death. AutoHCT recipients $<60$ years also avoided these outcomes, however, all allo-HCT recipients $>60$ years $(n=3)$ requiring MICU transfer died. Interestingly, being underweight (defined as $\mathrm{BMI} \leq 20$ ) rather than obesity was associated with a higher rate of MICU transfer. ISP use post alloHCT was not associated with poorer outcomes. Being on steroids at diagnosis of COVID-19 was significantly associated with an increased rate of MICU admission and mortality in the entire cohort (Supplementary 
Fig. 5). There was a trend toward increased mortality for the patients who had active GVHD at COVID-19 diagnosis $(p=0.09)$ (Supplementary Fig. 6). Patients with COVID-19 within the first year of alloHCT had poorer survival than that after the first year $(0 \%$ vs $87 \%$, respectively. $p=0.007)$. Patients with hemoglobin $<10 \mathrm{~g} / \mathrm{dL}$ or platelet count $<150\left(\times 10^{9} / \mathrm{L}\right)$ had significantly increased mortality (Supplementary Figs. 7 and 8). Other factors associated with poor outcomes included a higher $\mathrm{LDH}$, ferritin, and lymphopenia at COVID-19 diagnosis.

In conclusion, our study demonstrated that HCT recipients are at an increased risk of mortality compared to the general population [7]. Older age, being on steroids at diagnosis of COVID-19, and COVID-19 infection within 1 year of HCT, but not ISP use was associated with poor outcomes. Whether the steroid use by itself or comorbid conditions requiring steroid treatment is associated with inferior clinical outcomes deserves further investigation. Based upon our data, tapering or discontinuing ISP drugs in the setting of COVID-19 is not warranted.

Author contributions AV and CU: conceiving the idea, data collection, data analysis, literature search, writing and editing the manuscript. JH, UI, JM, SN, JM, DM, SK, KO, MB, KA: data collection, data analysis, literature search, writing and editing the manuscript.

\section{Compliance with ethical standards}

Conflict of interest The authors declare that they have no conflict of interest.
Publisher's note Springer Nature remains neutral with regard to jurisdictional claims in published maps and institutional affiliations.

\section{References}

1. He W, Chen L, Chen L, Yuan G, Fang Y, Chen W, et al. COVID19 in persons with haematological cancers. Leukemia. 2020;34:1637-45. http://www.nature.com/articles/s41375-0200836-7.

2. Hatzl S, Eisner F, Schilcher G, Kreuzer P, Gornicec M, Eller P, et al. Response to "COVID-19 in persons with haematological cancers." Leukemia. 2020. http://www.nature.com/articles/s41375020-0914-x.

3. Dhakal B, D'Souza A, Chhabra S, Hari P. Multiple myeloma and COVID-19. Leukemia. 2020;34:1961-3. http://www.nature.com/a rticles/s41375-020-0879-9.

4. van Doesum J, Chinea A, Pagliaro M, Pasquini MC, van Meerten T, Bakker M, et al. Clinical characteristics and outcome of SARSCoV-2-infected patients with haematological diseases: a retrospective case study in four hospitals in Italy, Spain and the Netherlands. Leukemia. 2020. http://www.nature.com/articles/s41375020-0960-4.

5. Kuderer NM, Choueiri TK, Shah DP, Shyr Y, Rubinstein SM, Rivera DR, et al. Clinical impact of COVID-19 on patients with cancer (CCC19): a cohort study. Lancet. 2020;395:1907-18. https://linkinghub.elsevier.com/retrieve/pii/S0140673620311879.

6. Zhang L, Zhu F, Xie L, Wang C, Wang J, Chen R, et al. Clinical characteristics of COVID-19-infected cancer patients: a retrospective case study in three hospitals within Wuhan, China. Ann Oncol. 2020;31:894-901. https://www.sciencedirect.com/science/a rticle/pii/S0923753420363833?via\%3Dihub.

7. Baud D, Qi X, Nielsen-Saines K, Musso D, Pomar L, Favre G. Real estimates of mortality following COVID-19 infection. Lancet Infect Dis. 2020;20:773. http://www.ncbi.nlm.nih.gov/pubmed/32171390. 